\title{
A Probabilistic Model for the Evolution of Porous Structure Caused by Solid-Phase Precipitation/Dissolution within Building Materials
}

\author{
Xiong Qing Xiang ${ }^{1, a}$ and Meftah Fekri, ${ }^{1, b}$ \\ ${ }^{1}$ Laboratory of Civil and Mechanical Engineering (LGCGM), National Institute for Applied \\ Sciences, Campus Beaulieu, 35700 Rennes, France \\ aqingxiang.xiong@insa-rennes.fr, ${ }^{\text {}}$ fekri.meftah@insa-rennes.fr
}

\begin{abstract}
Saline intrusion is a critical issue in building material because of the severe damages caused by the salt precipitation/dissolution process, especially for the porous material, which has good connectivity. When porous material is exposed to aggressive ambient, the pore structure, not only porosity but also pore size distribution, will be altered by salt precipitation/dissolution. As one of the most significant characteristics in the porous material, pore size distribution is always paid much attention in many literatures. However, a quantitative and practical determination method is still absent. This work aims to establish a probabilistic model to investigate the pore size distribution induced by solid-phase precipitation/dissolution. First, a lognormal distribution is proposed for the simulation of initial pore size distribution tested by the MIP method. Then we develop a probabilistic-based porous network to represent the evolution of microstructure due to precipitation/dissolution. To this end, two different transformation models are constructed to interpret the relation between initial pore radius and modified pore radius before and after precipitation/dissolution. With this probabilistic-based porous network, we could illustrate the precipitated profiles that evolve near the capillary interface during the salt precipitation/dissolution process for a given porosity and water saturation degree. Such a method could be used to interpret the mechanism of the local precipitation/dissolution process in pore scales, which cannot be implemented by experimental measurements.
\end{abstract}

Keywords: Pore Size Distribution, Precipitation/dissolution, Capillary Interface, Probabilistic Methods, Transformation Models.

\section{Introduction}

The durability of construction material is always prevalently focused research since it is strictly relevant to the long and durable service life of building structures. As is generally accepted, salt precipitation/dissolution is one of the main factors for the deterioration of construction material, especially for the porous construction material, which has highly connected cavity facilitating the transport of moisture, ions, and chemical fluxes (Huang et al., 2015). Salt precipitation/dissolution strongly influences the pore structure, which governs the most important properties of porous material, notably compressive strength for cement-based media (Cristina et al., 2012). For example, salt dissolution induces high porosity, which accelerates the permeability of gases and liquids within the pore structure, thus affects the compatibility and durability of the construction materials. As a result, it brings about high maintenance and repair costs. (Fenaux et al., 2019; Kumar et al., 2003). Therefore, the porous structure directly impacts the durability and resistance of construction material and components. More specifically speaking, research on the processes involved in salt-induced corrosion is of utmost benefit for assessing the durability of construction material.

Apart from the porosity, the pore size distribution (PSD) also plays a vital role in 
characterizing pore structure within porous media (Aligizaki et al., 2005). However, the porosity does not give any information about pore size, shape, distribution, and connectivity; thus, the PSD should be employed to give a precise depiction of pore structure. The PSD, which is so complicated that covers several orders of magnitude of pore size, is principally influenced by several aspects, for instance, water, air, chemical reactions, and other fluids. (JR Nimmo, 2013) It is essential to recognize that many efforts of experimental measurements have been devoted to the description of PSD by previous researchers (D.N. Winslow, 1968; S. Roels et al., 2001; E.Gallucci et al., 2007; Yanbin Yao et al., 2012; Gong Fuyuan et al., 2014). However, a quantitative and practical determination method is still absent. Since the pore structure is heterogeneous and anomalous morphology in pore sizes, it is hard to describe it in full detail by experimental measurements. Therefore, the most reasonable method is to assess it by probabilistic models.

In this work, we adopted a lognormal distribution to approach the initial PSD before solidphase precipitation occurred. Two representative models for pore radius were constructed by employing a monotonic transformation model and a non-monotonic transformation model. After several times of solid-phase precipitation, the new PSD was described by two different probabilistic methods. According to the comparison and parametric investigations, the proposed methods could provide us with an estimated prediction of the modified PSD, filling rate, and water saturation degree.

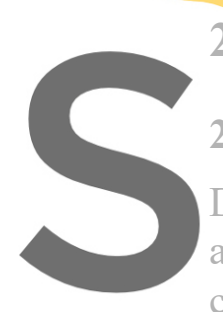

2 Methodologies

2.1 Transformation Models or Pore Radius
During the process of solid-phase precipitation/dissolution, the initial pore radius goes through
a multi-step of transformation with the evolution $r_{0} \rightarrow r_{1} \rightarrow \ldots \rightarrow r_{n} \rightarrow r_{n-1}$, which is
corresponding to the evolution of total porosity $\phi_{0} \rightarrow \phi_{1} \rightarrow \ldots \rightarrow \phi_{n} \rightarrow \phi_{n+1}$. According to the

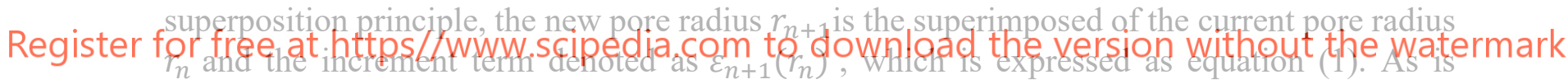
mentioned above, precipitation/dissolution occurs near the capillary interface. Thus, the waterfront $r_{c}$ is introduced into the transformation models of the pore radius. For the sake of simplification, it is assumed that the waterfront keeps constant as the evolution of pore structure. That is to say, the proposed pore system is open to the atmosphere to induce the water to evaporate into the ambient, which makes the waterfront be equilibrium. In this way, the capillary interface keeps constant for each time of transformation.

$$
r_{n+1}=f_{n+1}\left(r_{n}\right)=r_{n}+\varepsilon_{n+1}\left(r_{n}\right)
$$

Let us consider two different cases for the increment function $\varepsilon_{n+1}\left(r_{n}\right)$. For the monotonic model (see Figure 1a), water is filled in all the pores with the radius less than $r_{c}$. Solid-phase condenses inside the pores with the radius ranging from $r_{c}-\beta$ to $r_{c}+\beta$. When pore radius is large enough (i.e., more than $r_{c}+\beta$ ), the accumulated solid-phase is assumed to be constant as $\bar{\varepsilon}_{n+1}$. For the non-monotonic model (see Figure $1 \mathrm{~b}$ ), the precipitated solid only occurs near the waterfront with the range from $r_{c}-\beta$ to $r_{c}+\beta$; for large pores with radius more than $r_{c}+$ $\beta$, solid mass is supposed to be small enough to be neglected when compared to the large pore space. 

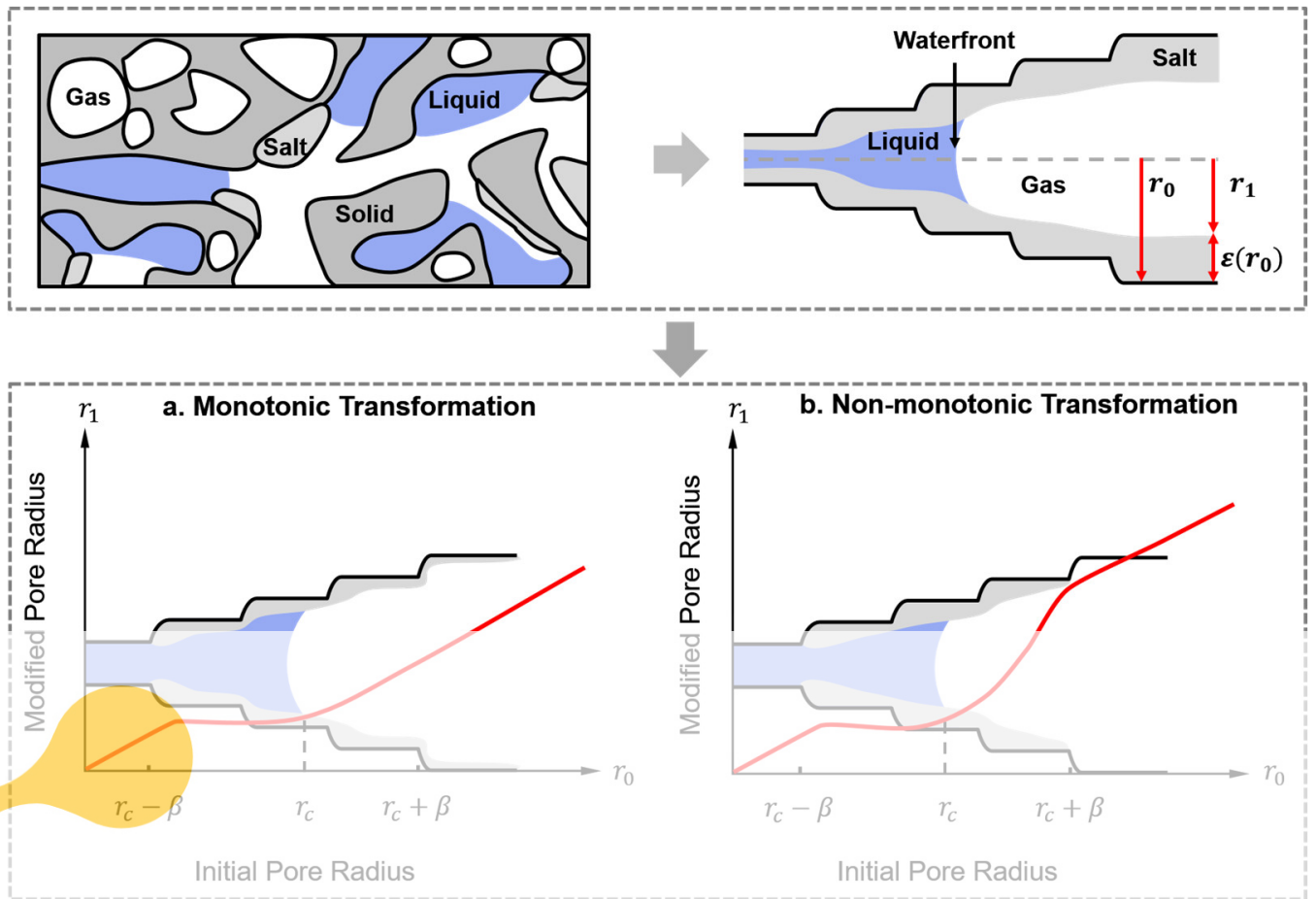

Figure 1. Schematic of the porous model for one single transformation $\left(r_{C}\right.$ is the maximum aperture filled with water, the so-called waterfront or capillary interface; $\beta$ is the opening zone, indicating the range of solid

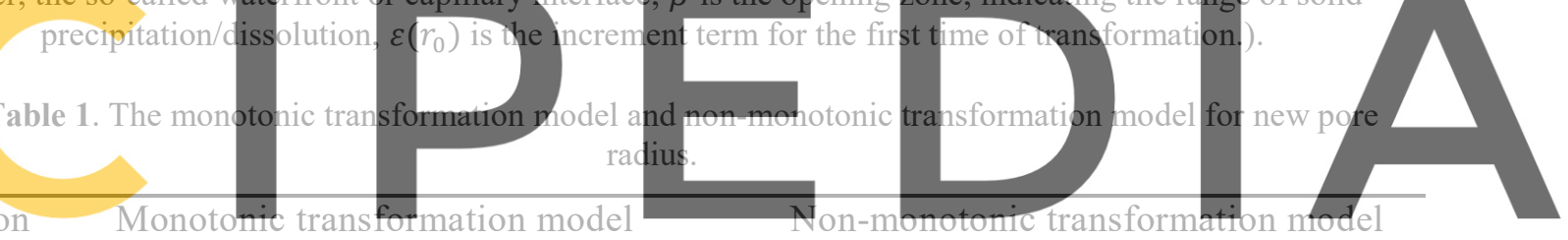

Function Monotonic trans formation model

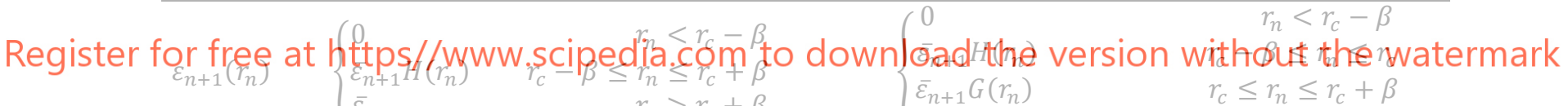

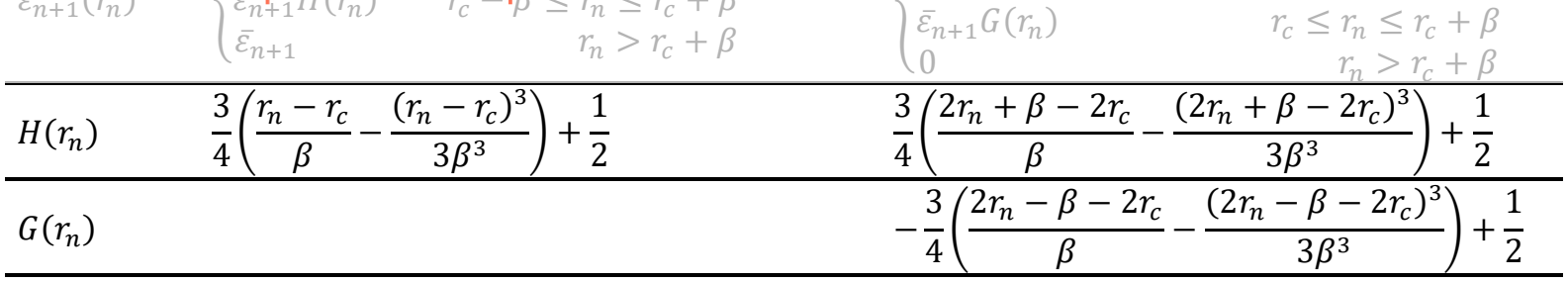

The derived function should

Remarks satisfy $H^{\prime}\left(r_{n}\right)>0$ in the case of salt precipitation, that is, $\bar{\varepsilon}_{n+1}$ is constrained in the range of $[0,-4 / 3 \beta]$

Table 1 shows the details of two different transformation functions. It should be noted if parameter $\bar{\varepsilon}_{n+1}>0$, salt dissolution occurs; if $\bar{\varepsilon}_{n+1}<0$, salt precipitation occurs. For the monotonic transformation model, the derived function $H^{\prime}\left(r_{n}\right)$ should be positive to make sure the transformation function is one-to-one. 


\subsection{Probabilistic Methods for Modified Pore Size Distribution}

The initial PSD for gel pores and capillary pores is presented in the form of lognormal probability density function that is expressed as:

$$
P_{0}\left(r_{0}\right)=\frac{1}{\sigma_{r} \sqrt{2 \pi} r_{0}} \exp \left(-\frac{\left(\ln r_{0}-\mu_{r}\right)^{2}}{2 \sigma_{r}^{2}}\right)
$$

where $\mu_{r}$, the location parameter, is the arithmetic mean of the representative pore size and $\sigma_{r}$, the standard deviation, is a measure of the dispersion of these pore size.

The total porosity for $\mathrm{n}+1$ th transformation is defined as:

$$
\phi_{n+1}\left(r_{n+1}\right)=\phi_{n+1} \int_{0}^{r_{n+1}} P_{n+1}(t) d t
$$

As we have the initial PDF $P_{0}\left(r_{0}\right)$ and two transformation models for pore radius, the new probability density function $P_{n+1}\left(r_{n+1}\right)$ for $n+1$ th transformation could be determined by the following two methods, which are presented in Table 2.

Table 2. Two probabilistic methods and their properties.

Method one Method two
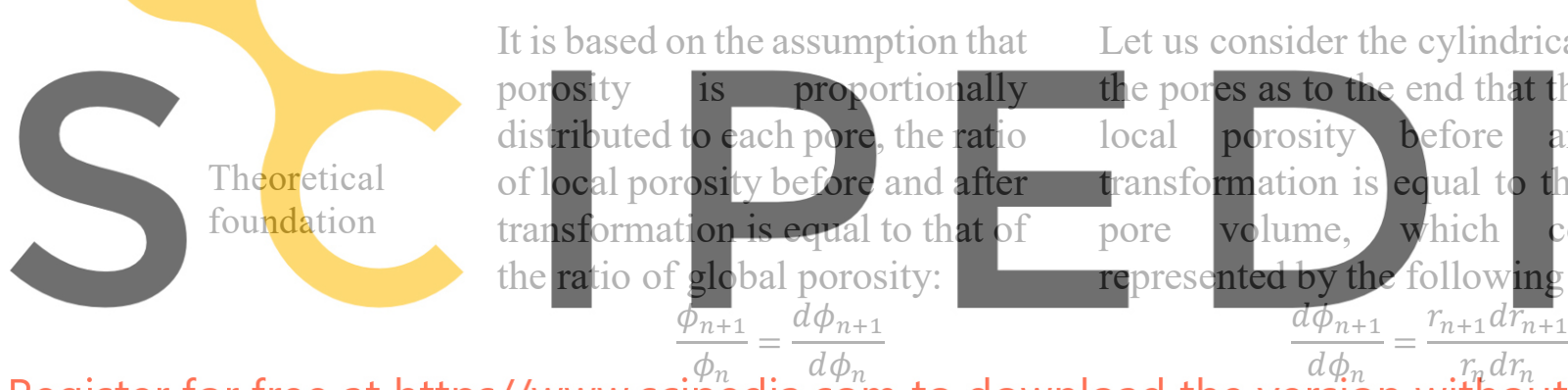

Let us consider the cylindrical form of

Theoretical

foundation

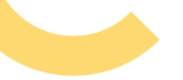

the ratio of

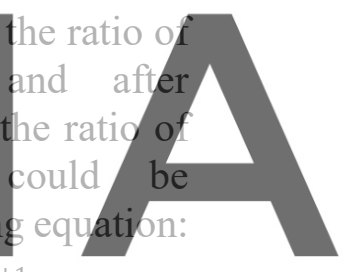

Register for free at https//www.scipedia.com to download the version without the watermark

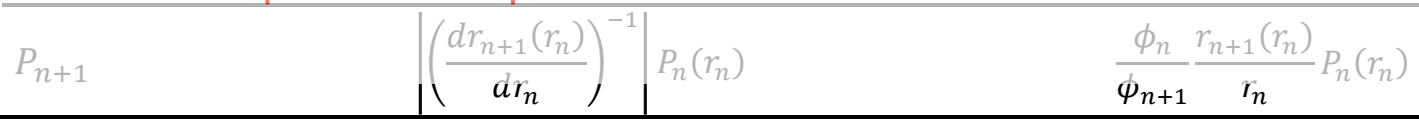

According to Figure 1, the capillary condensation occurs in pores with radii smaller than $r_{c}$, thus the water saturation degree $S_{l}(\mathrm{SD})$ can be obtained by the integration of the volume pores with sizes smaller than $r_{c}$.

$$
S_{l}=\int_{0}^{r_{c}} P_{n+1} d r_{n}
$$

\section{Implementation and Parametric Study}

The inputs parameters of initial pore size distribution were completed by experimental test mercury intrusion porosimetry (MIP) on masonry cement (see in Table 3 for more properties).

As the limitation of space, only the process of solid precipitation $\left(\bar{\varepsilon}_{n+1}<0\right)$ will be discussed in this work. The waterfront could occur in any position of capillary pores, ranging from $0.0025 \mu m$ to $5 \mu m$ (Huang et al., 2015), thus, the opening zone $\beta$ could be any value that 
is smaller than $r_{c}$.

Table 3. Characteristic parameters of lognormal distribution for the initial pore radius.

\begin{tabular}{ccccc}
\hline Initial porosity $\phi_{0}$ & Standard deviation $\sigma_{r}$ & Mean Value $\mu_{r}$ & $r_{\min }(\mu m)$ & $r_{\max }(\mu m)$ \\
\hline 0.20 & 1.2 & -1.8788 & 0.0015 & 165 \\
\hline
\end{tabular}

\subsection{One Transformation for the Monotonic Model}

Let us study one monotonic transformation of PSD evolves with four different capillary interfaces both by method one and method two. When solid-phase precipitation occurs, the porosity is assumed to decrease from 0.2 to 0.18 , and the opening zone is fixed at $0.05 \mu \mathrm{m}$. Then we can get the new PSD for the single transformation according to the equations in Table 1 and Table 2.

Figure 2 indicates that significant and sharp salt precipitation occurs near the waterfront for method one (see in Figure 2a). For method two (see in Figure 2b), the variation of pore radius is much smoother than method one. Moreover, the modification was distributed evenly to the whole range of pore size in method two. Thus, it can be concluded that for the porous materials sensitive to the capillary effect, such as the porous material with a large number of fine pores and high permeability, method one is more appropriate to describe the slight salt deposition process than method two. Of course, the extent of salt precipitation also depends on the type of

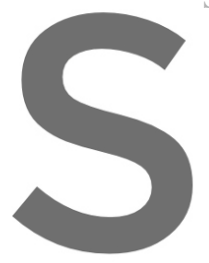
salts, temperature, concentration, and saturation degree of salt solution.

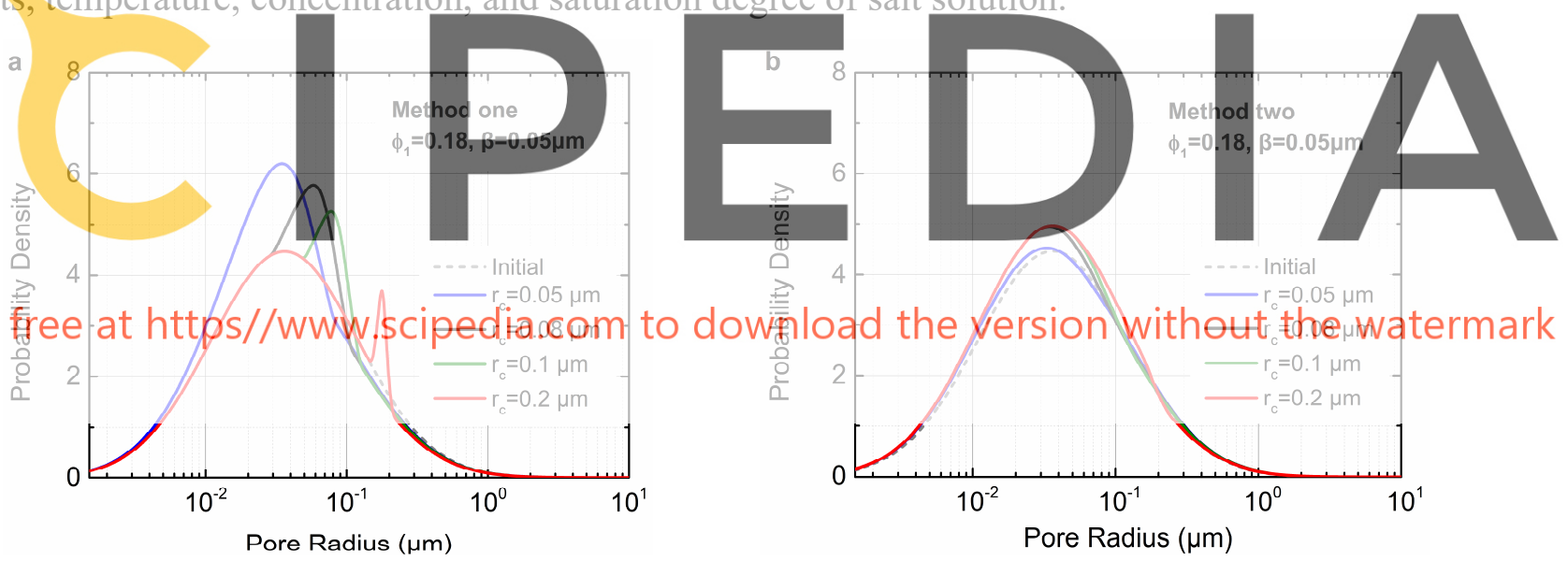

Figure 2. Pore size distribution with different waterfront for single transformation.

\subsection{Successive Transformations for the Non-Monotonic Model}

For the case of non-monotonic transformation models, we focus on five times of successive transformations with the porosity decreasing from 0.20 to 0.18 in the step of 0.004 . The waterfront is fixed at $0.1 \mu \mathrm{m}$, and the opening zone is valued at $0.08 \mu \mathrm{m}$. For non-monotonic transformation, there is no constraint to new porosity, and all we should concern about is that the critical parameter $\bar{\varepsilon}_{n+1}$ cannot make the new pore radius negative.

From Figure 3, we can find that both method one and method two generate a bimodal probability density as the porosity decreasing to a specific value. As is similar to the single transformation, the probability density presented by method one shows a noticeable increment 
of the peak value. In contrast, the probability density curve implemented by method two shows a slight shift to the left side, indicating produced more fine pores with the effect of precipitation.

As to the filling rate of pore radius due to the solid-phase precipitation presented in Figure 4 , by dividing $\varepsilon_{n+1}\left(r_{0}\right)$ with $r_{0}$, after one time of transformation, that of the method one is up to $30 \%$ while that of the method two accomplishes around $10 \%$. After five times of transformation, when the porosity decreases to 0.18 , the porous material described by method one has the filling rate of almost reaching $70 \%$, while method two only achieves over $30 \%$. Thus, at the same porosity, method one presents a more efficient filling rate of pore radius than method two.
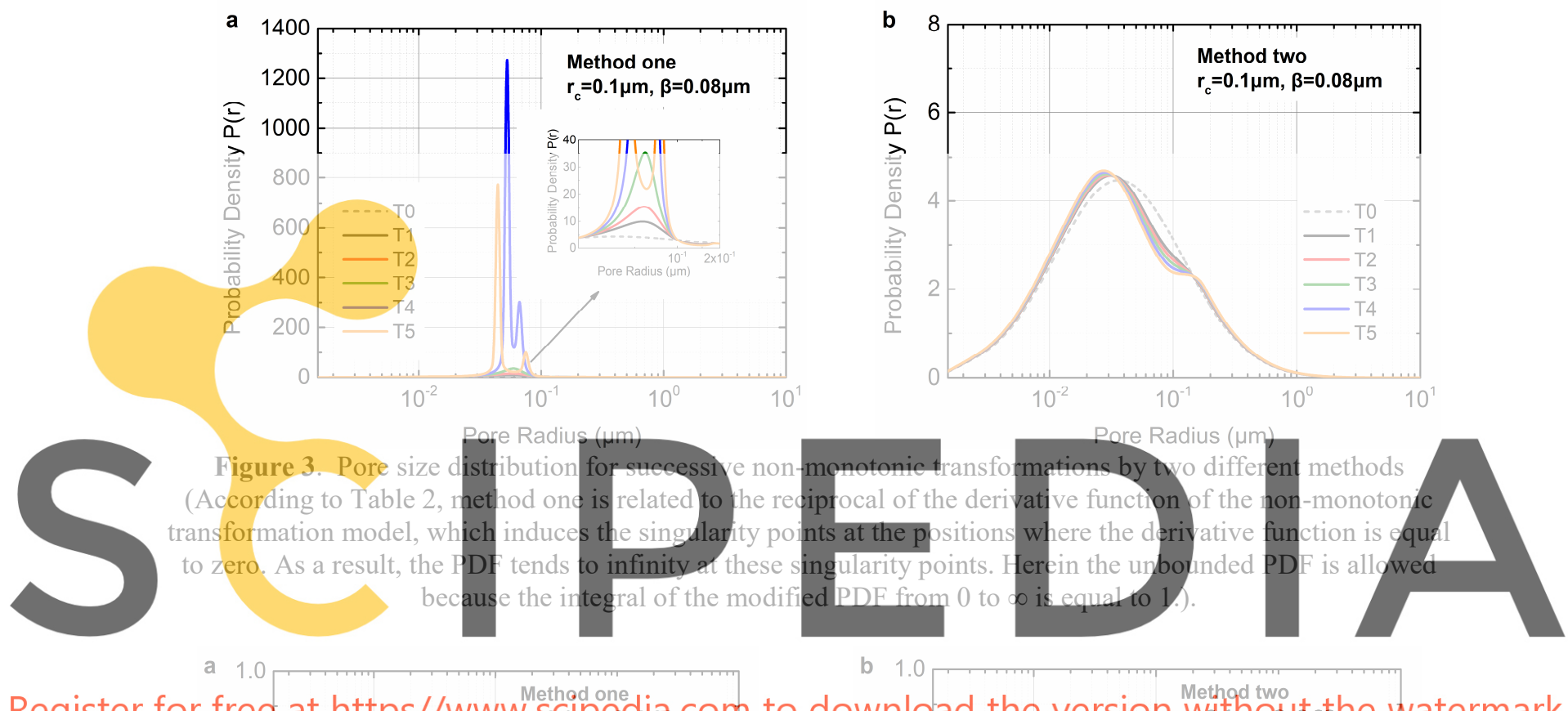

Register for free at https//WwW: Methad one
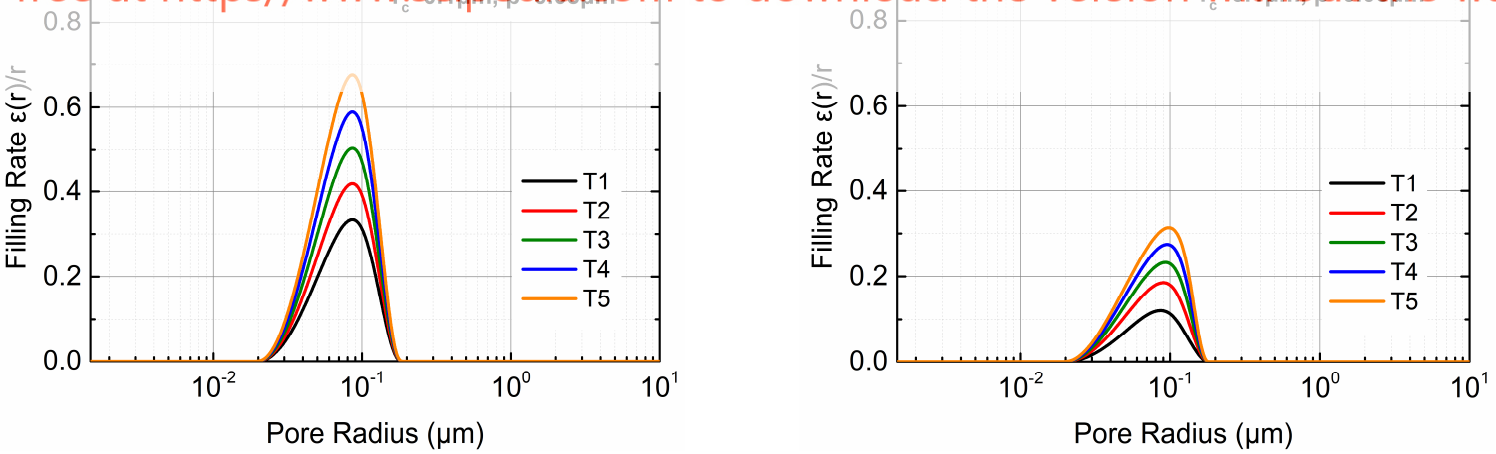

Figure 4. Filling rate of pore radius by two different methods during the process of salt precipitation.

To investigate the evolution of water saturation degree for successive transformations, we consider a case that porosity decreases from 0.2 to 0.185 by 30 steps, as is shown in Table 4 . 
Table 4. Parameter configurations for the investigation of the water saturation degree in non-monotonic successive transformations.

\begin{tabular}{cccccc}
\hline $\begin{array}{c}\text { Initial } \\
\text { porosity } \\
\phi_{0}\end{array}$ & $\begin{array}{c}\text { Final } \\
\text { porosity } \\
\phi_{30}\end{array}$ & $\begin{array}{c}\text { Transformation } \\
\text { Times }\end{array}$ & $\begin{array}{c}\text { Transformation } \\
\text { Steps of Porosity }\end{array}$ & $\begin{array}{c}\text { Waterfront } \\
r_{c}(\mu m)\end{array}$ & $\begin{array}{c}\text { Opening } \\
\text { Zone } \\
\beta(\mu m)\end{array}$ \\
\hline 0.2 & 0.185 & 30 & 0.0005 & 0.1 & 0.08 \\
\hline
\end{tabular}

Figure 5 shows the evolution of the water saturation degree with the porosity. It is observed that the water saturation degree calculated by method one shows a slight reduction while that is described by method two increases tremendously with the porosity decreasing. Besides, the incremental trend is more and more evident as the decreased porosity. It may be ascribed to the different filling rates of the two methods. For method two, the reduction rate of the pore volume is lower than that of water volume, leading to the increment of the water saturation degree. However, for method one, the reduction rate of the pore volume is fast enough to catch that of the water yolume, and the two rates are almost the same. Consequently, the water saturation degree by method two almost keeps constant.
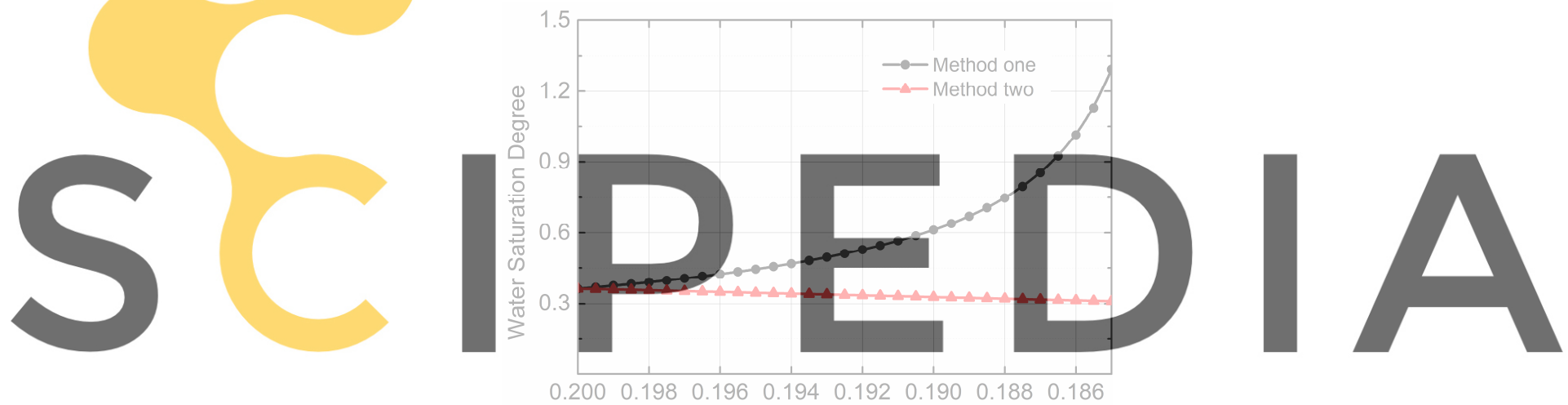

Register for free at https//www.scipedia.com to redownload the version without the watermark

Figure 5. Water saturation degree implemented by two methods in the case of salt precipitation.

\section{Conclusions}

- When the waterfront moves to large pores, method one shows more evident modification than method two for PSD in the process of salt precipitation. Moreover, method one is more appropriate to the porous material that is sensitive to the capillary effect than method two. Besides, the monotonic model is very limited used, which is only applicable to the slight salt precipitation.

- The existing two probabilistic methods could estimate the filling rate caused by salt precipitation. According to the simulated results of non-monotonic transformation models after five times of transformations, method one has a higher filling rate than method two, which indirectly leads to the stable water saturation degree in method one. Such an approach of method one allows the water condensation in the open system that does not depend on the evolution of the pore structure.

- For further investigation, proper consideration for the mobilization of waterfront in a closed system should be taken into account to fully understand the evolution of 
microstructure in the durability research for a porous material.

\section{Acknowledgement}

The author acknowledges Professor Ouali. Amiri of his generous providing experimental tested results for the reference of our work.

\section{ORCID}

Amiri, O: https://doi.org/10.1680/adcr.2005.17.1.39

Baroghel-Bouny Véronique: https://doi.org/10.1016/j.cemconres.2006.11.019

Fenaux, M : https://doi.org/10.1016/j.cemconcomp.2018.12.009

Gallucci Emmanuel: https://doi.org/10.1016/j.cemconres.2006.10.012

Gentilini Cristina : https://doi.org/10.1016/j.conbuildmat.2012.07.086

Gong Fuyuan: https://doi.org/10.1061/(ASCE)MT.1943-5533.0000945

Huang Qinghua: https://doi.org/10.1016/j.cemconres.2014.08.003

Kumar Rakesh: https://doi.org/10.1016/S0008-8846(02)00942-0

Nimmo, J: https://doi.org/10.1016/B978-0-12-409548-9.05265-9

Roels Staf: https://doi.org/10.1007/BF02481555

Windslow, D.N: https://doi.org/10.5703/1288284313747

YanbinYao: https://doi.org/10.1016/j.fuel.2011.12.039

References

Amiri, O., Ait-Mokhtar, A. and Sarhani, M. (2005). Tri-dimensional modelling of cementitious materials

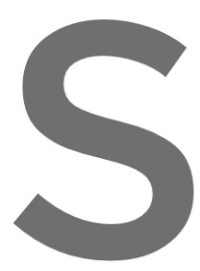
permeability from poly

in Cement Research,

Aligizaki, K. K. (2005).

\section{CRC Press.}

Fenaux, M., Reyes, E., Gálve in cement-based material
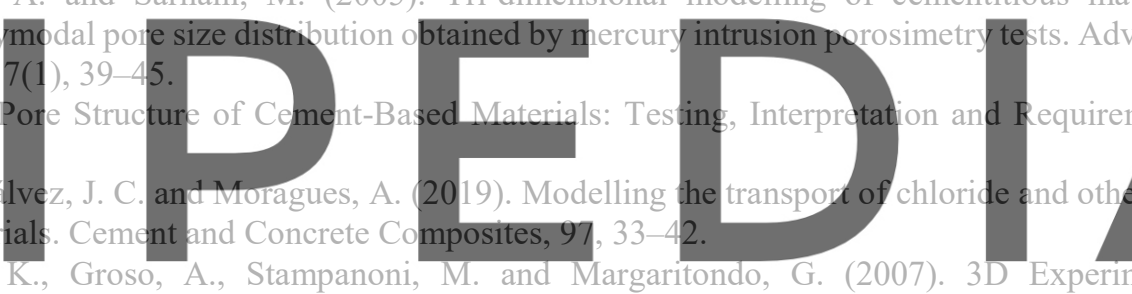

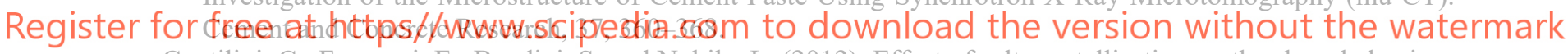

Gentilini, C., Franzoni, E., Bandini, S. and Nobile, L. (2012). Effect of salt crystallisation on the shear behaviour of masonry walls: An experimental study. Construction and Building Materials, 37, 181-189.

Gong Fuyuan, Zhang Dawei, Sicat Evdon, and Ueda Tamon. (2014). Empirical Estimation of Pore Size Distribution in Cement, Mortar, and Concrete. Journal of Materials in Civil Engineering, 26(7), 04014023.

Huang, Q., Jiang, Z., Gu, X., Zhang, W. and Guo, B. (2015). Numerical simulation of moisture transport in concrete based on a pore size distribution model. Cement and Concrete Research, 67, 31-43.

Kumar, R. and Bhattacharjee, B. (2003). Porosity, pore size distribution and in situ strength of concrete. Cement and Concrete Research, 33(1), 155-164.

Nimmo, J. (2013). Porosity and Pore Size Distribution. Reference Module in Earth Systems and Environmental Sciences.

Roels, S., Elsen, J., Carmeliet, J. and Hens, H. (2001). Characterisation of pore structure by combining mercury porosimetry and micrography. Materials and Structures, 34(2), 76-82.

Windslow, D.N. (1968). The Pore Size Distribution of Portland Cement Paste. JTRP Technical Reports.

Yao, Y. and Liu, D. (2012). Comparison of low-field NMR and mercury intrusion porosimetry in characterizing pore size distributions of coals. Fuel, 95, 152-158. 